\title{
In Vitro Comparison Of Fluoride Gel Alone and in Combination With Er,Cr:YSGG Laser on Reducing White Spot Lesions in Primary Teeth
}

\author{
Fatemeh Molaasadollah ${ }^{1}$, Mohammad Asnaashari' ${ }^{2}$, Fatemeh Mashhadi Abbas ${ }^{3}$, Maral Jafary ${ }^{1^{*}}$ \\ 1Department of Pediatric Dentistry, School of Dentistry, Shahid Beheshti University of Medical Sciences, Tehran, Iran \\ ${ }^{2}$ Department of Endodontics, School of Dentistry, Shahid Beheshti University of Medical Sciences, Tehran, Iran \\ ${ }^{3}$ Department of Oral \& Maxillofacial Pathology, School of Dentistry, Shahid Beheshti University of Medical Sciences, \\ Tehran, Iran
}

\section{*Correspondence to \\ Maral Jafary, Asistant Professor; Department of Pediatric Dentistry, School of Dentistry, Shahid Beheshti University of Medical Sciences, Tehran, Iran \\ Tel: +98 9127156795; \\ Fax: +98 2122749221; \\ Email:maraljafary.64@gmail.com}

Published online 27 September 2017

\begin{abstract}
Introduction: Laser therapy has been suggested as a method for caries-prevention, and ErCr:YSGG laser is increasingly used in dentistry. This study aimed to compare the efficacy of fluoride gel alone and in conjunction with Er,Cr:YSGG laser for remineralization of white spot lesions (WSLs) in primary teeth.

Methods: This study was conducted on 20 primary teeth with WSLs extracted for orthodontic reasons. Three sections were made of each tooth at the site of WSLs. The surface area of WSLs was measured under a stereomicroscope at $\times 25$ magnification. Samples were assigned to 3 groups of control (group 1), exposure to 1.23\% APF gel for 4 minutes (group 2) and $\mathrm{Er}, \mathrm{Cr}$ :YSGG laser (0.5 W power, $20 \mathrm{~Hz}$ frequency, $60 \%$ water, $40 \%$ air, pulse duration of $5 \pm 1$ seconds) plus fluoride (group 3). All samples were stored in artificial saliva for 10 days and then the surface area of the WSLs was measured again under a stereomicroscope. Data were analyzed using the Kruskal-Wallis, MannWhitney and Wilcoxon signed rank tests.

Results: The reduction in surface area of the WSLs was significantly different between the control and fluoride $(P<0.001)$ and also the control and laser plus fluoride groups $(P<0.001)$; the difference between fluoride and laser plus fluoride groups was not significant $(P=0.265)$. Comparison of the surface area of WSLs before and after the intervention showed no significant difference in group one $(P=0.737)$ while this difference in groups $2(P<0.001)$ and $3(P<0.001)$ was statistically significant.

Conclusion: The results showed that Er,Cr:YSGG laser irradiation plus 1.23\% APF gel was not significantly different from the application of fluoride gel alone in enhancing the remineralization of WSLs.

Keywords: White spot lesions; Fluoride; Er,Cr:YSGG laser; APF gel; Remineralization.
\end{abstract}

\section{Introduction}

Despite the advances in caries prevention in permanent teeth, preventive methods have not been very efficient for primary teeth. ${ }^{1}$ In smooth surfaces of teeth, caries mainly occurs at plaque accumulation areas and is initially in the form of white spots or lines. ${ }^{2}$ At this stage, caries is reversible and the process of demineralization can be stopped and replaced by remineralization. ${ }^{3}$ For this purpose, fluoride-containing products are used as the standard method of caries prevention. Laser has also been evaluated for this purpose in some previous studies. Laser causes chemical alterations in the enamel and increases its resistance to caries. ${ }^{4,5}$

Erbium lasers in 2 common forms of Er:YAG and Er,Cr:YSGG are popular in dentistry. They have similar characteristics and both use solid crystals as conductors.
The erbium ion $\left(\mathrm{Er}^{3+}\right)$ is the active ion in these lasers. ${ }^{6,7}$ Er,Cr:YSGG laser contains erbium and chromium ions, yttrium crystals, scandium, gallium and garnet. It operates at $78.2 \mu \mathrm{m}$ wavelength for dental purposes such as cavity preparation, caries removal, endodontic treatment and surgery. This wavelength is well absorbed by the $\mathrm{OH}^{-}$groups present in hydroxyapatite crystals and increases the temperature of tooth surface up to $800^{\circ} \mathrm{C}$ at the ablation threshold and results in crystallographic changes in the enamel without causing evaporation or melting of carbonated hydroxyapatite. ${ }^{8-11}$

Simultaneous application of fluoride and laser as a caries prevention method has been extensively evaluated in previous studies. Some studies have reported that application of both methods is beneficial and have shown that fluoride uptake increases after laser irradiation. ${ }^{12-17}$ 
Some other studies have shown that simultaneous application of laser and fluoride has no advantage over the use of each of them alone to increase acid resistance. ${ }^{18,19}$ However, controversy exists regarding the application of fluoride before or after laser therapy. ${ }^{20}$ Also, most of these studies have been conducted on permanent teeth and those on primary teeth are scarce. ${ }^{21-23}$

Some previous studies have artificially induced initial carious lesions and assessed the effect of different preventive measures on them. ${ }^{11,18,20,23,24}$

In order to simulate oral clinical conditions, primary teeth with white spot lesions (WSLs) were used in the current study. This study aimed to compare the efficacy of fluoride gel alone and in conjunction with Er,Cr:YSGG laser for remineralization of WSLs in primary teeth.

\section{Methods}

Twenty primary teeth with sound crowns and no caries were extracted due to over-retention, ankylosis or space deficiency and stored in saline. The teeth were then dried with air spray and visually inspected for absence of noncarious WSLs such as hypoplasia, hypomineralization and fluorosis and the teeth with WSLs due to caries were entered in the study. At least 2 adequately large WSLs or white spot lines were present on teeth surfaces. The samples were disinfected using $0.5 \%$ chloramine $\mathrm{T}$ solution. WSLs were cut in half by a diamond disc (Huaxian Gaoping YanaDa Diamond, China) under water coolant in occlusogingival direction towards the pulp. If the lesion was adequately large, it was divided into 3 sections; otherwise, another WSL on the surface was sectioned. In total, 3 slices of each tooth were obtained (Figure 1). The entire surface of specimens except for the WSL was covered with nail varnish. The specimens were then evaluated under a stereomicroscope (Olympus, Japan) by a blind observer (Figure 2). Specimens in which demineralization had reached dentinoenamel junction (DEJ) and caries was seen as a dark line at the DEJ were excluded along with the other 2 specimens obtained from the same teeth and replaced with new specimens.

Three sections obtained of each tooth were randomly assigned to one of the 3 groups:

Group 1 or control group: no intervention.

Group 2: $1.23 \%$ APF gel was applied on the surface of specimens for 4 minutes.

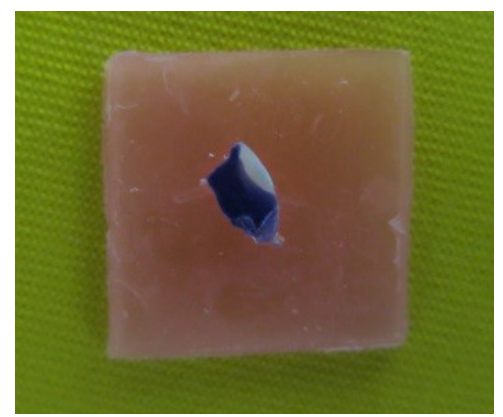

Figure 1. Slice of Tooth With White Spot Lesion.
Group 3: Specimens were subjected to Er,Cr:YSGG laser irradiation followed by the application of $1.23 \%$ APF gel. The specimens were coded from 1 to 60 with a black nail varnish. Digital images were obtained of each specimen using a camera attached to a stereomicroscope at $\times 25$ magnification. Digital images were stored in a computer attached to a camera. Using HYCAD version 5.35 software, the surface area of the WSLs was measured and recorded.

After recording the baseline data, the interventions were performed. In the control group, no intervention was performed on the specimen. In group 2, 1.23\% APF gel (60 Taste ${ }^{\varpi}$ Second Fluoride Gel, Pascal Company Inc., USA) was applied to the specimens for 4 minutes and was then wiped off with a wet gauze.

In group 3, specimens were first mounted in acrylic resin and Er,Cr:YSGG laser was irradiated (Waterlase, Biolase Technology, San Clemente, CA, USA) on the lesion (0.5 W power, $20 \mathrm{~Hz}$ frequency, $60 \%$ water, $40 \%$ air, pulse duration of $5 \pm 1$ seconds).

Duration of irradiation was determined based on the protocols used in previous studies and conduction of a pilot study taking into account the surface area of the WSLs. Immediately after laser irradiation, each specimen was exposed to fluoride gel as described earlier. All samples were immersed in artificial saliva (Kin Hidrat, Laboratorios, Spain). Due to the presence of fluoride in remineralizing solutions, which are used for $\mathrm{pH}$ cycling), ${ }^{25}$ these solutions were not used in our study. Moreover, in order to decrease the confounding effect of fluoride released from the specimens into the artificial saliva and to standardize the samples, all samples were immersed in the same solution altogether. After 10 days, the samples were observed under a stereomicroscope again with the same magnification and digital images were captured by the same examiner. Surface area of the lesions after the intervention was measured using HYCAD software. These values were compared with the baseline values before the intervention.

Data were expressed as the mean, standard deviation (SD), maximum and minimum values. SPSS version 16 software (SPSS Inc., Chicago, IL, USA) was used for statistical analysis and drawing tables and Excel XP software was employed for drawing the diagrams. The Kruskal-Wallis, Mann-Whitney and Wilcoxon signed

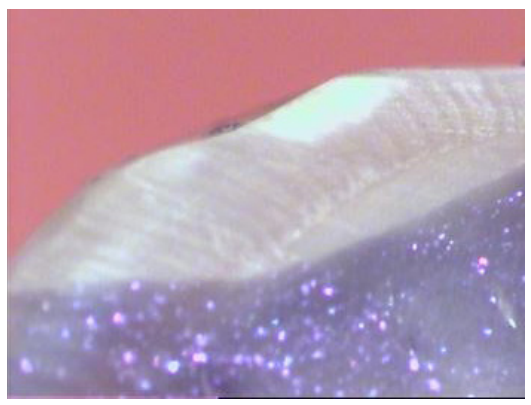

Figure 2. White Spot Lesion Under Stereomicroscope. 
rank tests were used for data analysis. HYCAD software was used to compare the changes in the surface area of WSLs of primary teeth following the application of fluoride alone and in combination with Er,Cr,YSGG laser on stereomicroscopic images. The obtained values were entered into SPSS version 16 software.

\section{Results}

The mean, SD, minimum and maximum surface area of the WSLs before and after the interventions are shown in Table 1.

The Shapiro-Wilk test showed that the variable of change in surface area of WSLs in groups did not have a normal distribution (maximum possibility of 0.037 ) and Levene test showed inequality of variances in the groups. Thus, to compare the changes in the surface area of WSLs in the groups, non-parametric Kruskal-Wallis test was employed and revealed a significant difference among the 3 groups $(P<0.001$; Table 2$)$.

Pairwise comparison of groups using the Mann-Whitney $\mathrm{U}$ test showed that the reduction in WSL surface area was significantly different between groups one and 2 $(P<0.001)$ and groups one and $3(P<0.001)$; however, no significant difference was noted between groups 2 and 3 $(P=0.265)$ in this regard.

Comparison of the surface area of WSLs before and after the intervention in each group showed no significant difference in this regard in group one $(P=0.737)$; however, groups 2 and 3 showed significant differences after the intervention compared to baseline (both $P$ values $<0.001$ ) (Table 3; Figure 3).

\section{Discussion}

Despite a general reduction in caries prevalence in primary teeth in the recent years, its prevalence in some specific populations has increased. Early childhood caries (ECC) is still a major public health dilemma. Considering the high cost of dental treatments and difficulties in behavior management of children, greater attention to prevention of caries seems imperative.

Optimal efficacy of professional application of topical fluoride gel for prevention of caries and reversing the process of demineralization in WSLs has been confirmed. ${ }^{7,26}$

Considering the young age of children at the onset of
ECC and limitations in frequent applications and use of high dose fluoride at early ages (due to its side effects), ${ }^{1,26}$ strategies must be adopted to reinforce the effects of topical fluoride application while preventing its side effects. Laser application has been recommended for this purpose and its use with/without fluoride has been proposed by some researchers. ${ }^{12-18}$ Several mechanisms have been attributed to laser. Laser decreases the content of carbonate ions and thus, decreases the solubility of enamel, changes the polarization of enamel components and increases the substantivity of fluoride ions next to the enamel. Laser re-crystalizes and fuses the enamel prisms and by changing the organic structure of enamel, decreases its acid solubility and confers caries resistance to tooth structure. ${ }^{27-29}$ However, since the application of laser requires costly equipment, ${ }^{7}$ the possibility of laser enhancing the preventive effect of fluoride must be carefully investigated considering the above-mentioned limitations of laser. The current in vitro, experimental study compared the reduction in surface area of 60 WSLs on the crowns of 20 extracted primary teeth in 3 groups of control, 1.23\% APF gel and Er,Cr,YSGG laser in combination with $1.23 \%$ APF gel.

In most previous studies, WSLs were not present on the teeth surfaces in the first place and incipient caries were later induced artificially via demineralization and remineralization cycles. ${ }^{11-13,18,20,21,30,33}$

The current study results showed that in the nointervention control group, where the samples were only immersed in artificial saliva, no change occurred

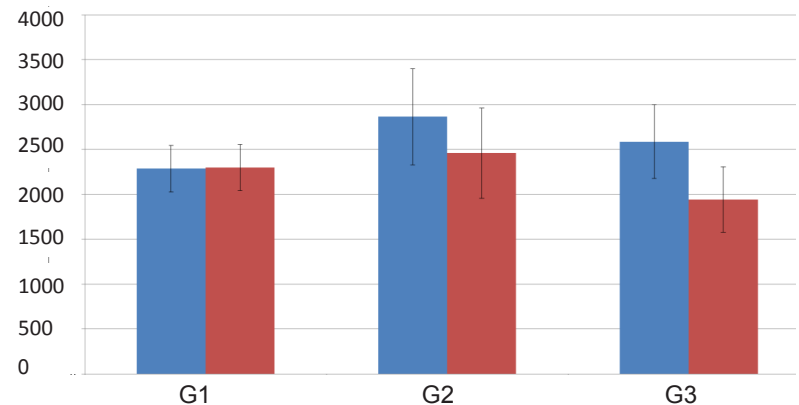

Figure 3. Changes in the Surface Area of WSLs After the Intervention in the 3 Groups.

G1: Control; G2: Fluoride; G3: Laser+ fluoride; Blue: Before the intervention; Red: After the intervention.

Table 1. The Mean, Standard Deviation, Minimum and Maximum Surface Area of the WSLs Before and After the Interventions

\begin{tabular}{|c|c|c|c|c|c|c|c|}
\hline Descriptives & & Number & Median & Standard Deviation & Maximum & Maximum & Mean \\
\hline \multirow{2}{*}{ Before treatment } & G1 & 20 & 2285.7700 & 260.69454 & 362.05 & 4435.77 & 2286.4785 \\
\hline & G2 & 20 & 2863.8765 & 535.77088 & 964.88 & 8711.75 & 1839.3085 \\
\hline \multirow{3}{*}{ After treatment } & G3 & 20 & 2586.3360 & 411.85628 & 902.28 & 8429.26 & 2178.6800 \\
\hline & G1 & 20 & 2300.8432 & 256.27244 & 375.06 & 4262.98 & 2450.5270 \\
\hline & G2 & 20 & 2458.4586 & 501.10941 & 524.56 & 7520.94 & 1597.3565 \\
\hline \multirow{3}{*}{ Difference } & G3 & 20 & 1939.9379 & 365.06409 & 707.21 & 7515.67 & 1306.5945 \\
\hline & G1 & 20 & 15.0732 & 33.11909 & -216.70 & 347.95 & -14.5200 \\
\hline & G2 & 20 & -405.4180 & 81.25373 & -1190.82 & -52.93 & -247.4705 \\
\hline
\end{tabular}


Table 2. Comparison of the 3 Groups Using the Kruskal-Wallis Test

\begin{tabular}{lll}
\hline Group & Number & Mean Value \\
\hline G1 & 20 & 48.20 \\
G2 & 20 & 23.65 \\
G3 & 20 & 19.65 \\
Total & 60 & \\
\hline
\end{tabular}

Chi-square $=31.340, d f=2$, Asymp. Sig. $=0.000$.

Table 3. Comparison of the Lesions Before and After the Intervention Using Wilcoxon Signed Rank Test

\begin{tabular}{lc}
\hline Group & Before and After the Intervention \\
\hline 1 & -.336 \\
Asymp. Sig. (2-tailed) & .737 \\
2 & -3.920 \\
Asymp. Sig. (2-tailed) & .000 \\
3 & -3.920 \\
Asymp. Sig. (2-tailed) & .000 \\
\hline
\end{tabular}

in the extent of lesions. In group 2, where the samples were exposed to $1.23 \%$ APF gel for 4 minutes and were then immersed in artificial saliva, the extent of lesions significantly decreased after the intervention compared to the baseline value $(P<0.001)$. In group 3 , where laser was irradiated prior to fluoride application, a significant reduction occurred in the extent of lesions $(P<0.001)$. Groups 2 and 3 showed significant differences with the control group $(\mathrm{P}<0.001)$ but the difference between the fluoride and laser plus fluoride group was not significant $(P=0.265)$.

Azevedo et al, in 2012 evaluated the enamel resistance of primary teeth to acid using microhardness test and assessed the demineralization depth under a microscope. They found higher resistance in APF and APF plus laser groups compared to the control group but the APF and APF plus laser groups were not significantly different; this finding is in line with our results. They used Nd:YAG laser in their study. Moreover, the samples were initially sound and were then subjected to demineralization/ remineralization cycles. ${ }^{33}$

Tagliaferro et al assessed primary enamel resistance to demineralization by the Knoop microhardness test. The enamel resistance in groups treated with laser and/or APF was higher compared to the control group, and combined application of laser and fluoride caused no significant change in the results compared to other interventions, which is similar to the current study results. They artificially created caries-like lesions on primary teeth and applied laser and fluoride on the lesions, simulating oral environment, which is similar to our study. However, their study was different from ours in that after the application of laser and fluoride in their study, the specimens were subjected again to $\mathrm{pH}$ cycling. Moreover, WSLs were artificially created in their study and they used $\mathrm{CO}_{2}$ laser. ${ }^{30}$ Hicks et al in 1995 assessed the depth of WSLs under a microscope and showed that application of APF before and after laser irradiation increased the enamel resistance compared to the control group. These results are in agreement with our findings. Their study, however, had some differences with ours. For instance, they used argon laser in their study and did not evaluate the application of APF gel alone. Moreover, they artificially induced the WSL after the application of laser and APF gel. ${ }^{22}$

Westerman et al in 2004 assessed the primary enamel resistance by measuring the depth of WSLs under a microscope and showed that application of laser along with APF gel decreased the depth of WSLs compared to the control group. They did not evaluate the efficacy of application of fluoride alone. They used argon laser and induced caries-like lesions artificially following the application of laser and fluoride. ${ }^{21}$

Anaraki et al in 2012 assessed enamel resistance of molar teeth using atomic absorption spectroscopy and showed that demineralization in the $\mathrm{CO}_{2}$ laser group was lower than that in APF and APF plus Er,Cr,YSGG laser groups. They showed that CO2 laser significantly enhanced enamel resistance to demineralization compared to the application of APF alone. However, no difference was noted in enamel resistance following the application of $\mathrm{Er}, \mathrm{CR}, \mathrm{YSGG}$ laser plus fluoride and APF alone, which is in accordance with our results. ${ }^{13}$

Ana et al in 2012 used the Knoop microhardness test and noted no difference in the application of Er,Cr,YSGG laser plus APF compared to the use of APF gel alone, which is in agreement with the current study results. However, measurement of calcium fluoride showed higher amount of calcium fluoride in combined use of laser and fluoride compared to the use of fluoride alone. As explained earlier, this can be attributed to the effect of laser on maintaining the fluoride ions next to the enamel by changing enamel polarization. But, this change did not result in a significant increase in enamel microhardness. ${ }^{11}$ Chin et al in 2004 used secondary ion mass spectrometry and showed that 2 and $4 \mathrm{~W} \mathrm{CO}_{2}$ laser enhanced the preservation of fluoride ions adjacent to the enamel. ${ }^{34}$

Moslemi et al in 2009 used atomic absorption spectrometry and showed that permanent enamel had higher resistance to demineralization following the use of Er,Cr,YSGG laser combined with APF compared to the use of APF alone; this finding is in contrast to our results. It should be noted that method of assessment of enamel resistance in their study was different from ours and they assessed enamel demineralization by measuring the amount of ions released from the enamel into the solution; whereas in our study, remineralization of lesions was observed under a microscope as a criterion of enamel resistance. Application of laser plus fluoride caused no significant change in comparison with the use of fluoride alone in our study; but, as seen in Figure 3, laser combined with fluoride increased enamel resistance to a greater extent, although it did not reach statistical significance (by observation of the extent of lesions under a microscope). This difference in results may be attributed to different methods of assessment of enamel resistance in the 2 studies. $^{20}$

Controversy exists regarding the sequence and order of application of laser and fluoride in different studies. 
Considering the theory that laser application results in maintenance of fluoride ions next to the enamel, in the current study, samples in laser plus fluoride group were exposed to APF gel immediately after laser irradiation. In studies conducted by Bevilácqua et $\mathrm{al}^{31}$ and Ana et $\mathrm{al},{ }^{11}$ fluoride was applied following laser irradiation. In the study by Bevilácqua et $\mathrm{al},{ }^{31}$ enamel resistance following the application of APF and laser was higher than that in the control group but they did not apply APF gel alone. ${ }^{29}$ In the study by Ana et al, Er,CR,YSGG laser plus APF caused no significant difference as compared to the use of APF gel alone. ${ }^{11}$

Moslemi et al, ${ }^{20}$ Altinok et al, ${ }^{18}$ Westerman et al, ${ }^{21}$ Hicks et $\mathrm{al}^{, 22}$ and Tagliaferro et $\mathrm{al}^{30}$ compared the application of laser followed by fluoride with the use of fluoride followed by laser irradiation and found no significant difference in the results.

Based on the results of the above-mentioned studies, it seems that the sequence and order of application of laser and fluoride does not have any significant effect on the results, and the differences in the results of studies is probably attributed to other factors such as laser properties and different assessment methods of enamel resistance.

Most previous studies have investigated the enamel resistance following the application of fluoride and laser and conduction of $\mathrm{pH}$ cycling or use of demineralizing solutions. ${ }^{8,11-13,18,20-22,28,30-32,34}$

Tagliaferro et al, ${ }^{30}$ first artificially induced caries-like lesions on the teeth (in contrast to our study) and then similar to the current study, applied laser and fluoride gel on the lesions, simulating the oral environment. In our study, the process of remineralization of WSLs naturally formed in the oral environment was evaluated in order to better simulate in vivo conditions.

For the purpose of remineralization and providing the calcium and phosphate ions required for this process and also to better simulate the oral clinical setting, specimens in our study were immersed in artificial saliva for 10 days, which contained these ions. Remineralizing solutions used for $\mathrm{pH}$ cycling contain fluoride $\mathrm{e}^{25}$ and thus, were not used in our study.

As stated earlier, most previous studies artificially created the lesions while we used naturally formed WSLs in the oral cavity. This can affect the methodology of the study and consequently the results.

\section{Conclusion}

The results of this study confirm the efficacy of fluoride for decreasing the extent of WSLs in vitro. Also, the results showed that application of laser (with the exposure settings and the sequence described earlier) did not reinforce the efficacy of fluoride for decreasing the extent of WSLs.

\section{Ethical Considerations}

Ethical considerations were approved by Shahid Beheshti
University of Medical Sciences Ethics Committee and the Code No. is IR.SBMU.REC.1393.517.

\section{Conflict of Interests}

The authors declare that there is no conflict of interest.

\section{References}

1. McDonald RE AD, Dean JA. Dentistry for the Child and Adolescent. 8th ed. USA: Mosby, Elsevier; 2004.

2. Gutknecht NF, Franzenb R, Vanweersch L, Lampert F. Lasers in pediatric dentistry - a review. J Oral Laser Appl. 2005;5(4):207-218

3. Guzmán-Armstrong S, Chalmers J, Warren JJ. White spot lesions: prevention and treatment. Am J Orthod Dentofacial Orthop. 2010;138(65):690-696. doi:10.1016/j. ajodo.2010.07.007.

4. Contreras-Bulnes R, Olea-Mejía OF, Rodríguez-Vilchis LE, Scougall-Vilchis RJ, Centeno-Pedraza C. Structural Changes on Human Dental Enamel Treated with Er:YAG, CO2 Lasers and Remineralizing Solution: EDS Analysis. In: Virdi M, ed. Oral Health Care - Prosthodontics, Periodontology, Biology, Research and Systemic Conditions. InTech; 2012:209-318.

5. Lussi A, Hellwig E, Klimek J. Fluorides -mode of action and recommendation for use. Schweiz Monatsschr Zahnmed. 2012;122(11):1030-42.

6. Diaci J, Gaspirc B. Comparison of Er:YAG and Er,Cr:YSGG lasers used in dentistry. J Laser Health Acad. 2012;1(1):113.

7. Verma SK, Maheshwari S, Singh RK, Chaudhari PK. Laser in dentistry: an innovative tool in modern dental practice. Natl J Maxillofac Surg. 2012;3(2):124-32. doi: 10.4103/09755950.111342.

8. Apel C, Meister J, Schmitt N, Graber HG, Gutknecht N. Calcium solubility of dental enamel following sub-ablative Er:YAG and Er:YSGG laser irradiation in vitro. Lasers Surg Med. 2002;30(5):337-341.

9. Dorland's Illustrated Medical Dictionary. 32nd ed. United USA: Elsvier; 2011:1007.

10. Van AG. Erbium lasers in dentistry. Dent Clin North Am. 2004;48(4):1017-1059.

11. Ana PA, Tabchoury CP, Cury JA, Zezell DM. Effect of Er,Cr:YSGG laser and professional fluoride application on enamel demineralization and on fluoride retention. Caries Res. 2012;46(5):441-451. doi:10.1159/000333603.

12. Fekrazad R, Ebrahimpour L. Evaluation of acquired acid resistance of enamel surrounding orthodontic brackets irradiated by laser and fluoride application. Lasers Med Sci. 2013;29(6):1793-1798. doi:10.1007/s10103-013-1328-z.

13. Anaraki SN, Serajzadeh M, Fekrazad R. Effects of laser-assisted fluoride therapy with a $\mathrm{CO} 2$ laser and Er, Cr:YSGG laser on enamel demineralization. Pediatr Dent. 2012;34(4):e92-e96.

14. Sobral MA, Lachowski KM, de Rossi W, Braga SR, Ramalho KM. Effect of Nd:YAG laser and acidulated phosphate fluoride on bovine and human enamel submitted to erosion/abrasionor erosion only: an in vitro preliminary study. Photomed Laser Surg. 2009;27(5):709-713.

15. Hsu CY, Jordan TH, Dederich DN, Wefel JS. Laser-matrixfluoride effects on enamel demineralization. J Dent Res. 2001;80(9):1797-1801.

16. Rios D, Magalhaes $\mathrm{AC}$, Machado MA, et al. In vitro 
evaluation of enamel erosion after Nd:YAG laser irradiation and fluoride application. Photomed Laser Surg. 2009;27(5):743-747. doi:10.1089/pho.2008.2384.

17. Liu Y, Hsu CY, Teo CM, Teoh SH. Potential mechanism for the laser-fluoride effect on enamel demineralization. J Dent Res. 2013;92(1):71-75. doi:10.1177/0022034512466412.

18. Altinok B, Tanboga I, Peker S, Eren F, Bakkal M, Peker F. The effect of laser-activated acidulated phosphate fluoride on enamel submitted to erosive solution only: an in vitro preliminary evaluation. Eur J Paediatr Dent. 2011;12(1):1316.

19. Chen CC, Huang ST. The effects of lasers andfluoride on the acid resistance of decalcified human enamel. Photomed Laser Surg. 2009;27(3):447-452.

20. Moslemi M, Fekrazad R, Tadayon N, Ghorbani M, Torabzadeh H, Shadkar MM. Effects of ER,Cr:YSGG laser irradiation and fluoride treatment on acid resistance of the enamel. Pediatr Dent. 2009;31(5):409-413.

21. Westerman GH, Hicks MJ, Flaitz CM, Ellis RW, Powell GL. Argon laser irradiation and fluoride treatment effects on caries-like enamel lesion formation in primary teeth: an in vitro study. Am J Dent. 2004;17(4):241-244.

22. Hicks J, Flaitz C, Ellis R, Westerman G, Powell L. Primary tooth enamel surface topography with in vitro argon laser irradiation alone andcombined fluoride and argon laser treatment: scanning electon microscopic study. Pediatr Dent. 2003;25(5):491-496.

23. Santaella MR, Braun A, Matson E, Frentzen M. Effect of diode laser and fluoride varnish on initial surface demineralization of primary dentition enamel: an in vitro study. Int J Paediatr Dent. 2004;14(3):199-203.

24. Manesh SK, Darling CL, Fried D. Nondestructive assessment of dentin demineralization using polarizationsensitive optical coherence tomography after exposure to fluoride and laser irradiation. J Biomed Mate Res. 2009;90(2):802-812.

25. Ab-Ghani Z, Ngo H, McIntyre J. Effect of remineralization/ demineralization cycles on mineral profiles of Fuji IX Fast in vitro using electron probe microanalysis. Aust Dent J. 2007;52(4):276-281.

26. Harris NO, Garcia-Godoy F, Nathe CN. Primary Preventive Dentistry. 7th ed. Upper Saddle River, NJ: Pearson; 2009.

27. Raucci-Neto W, de Castro-RaucciLM, Lepri CP, FaraoniRomano JJ, da Silva JM, Palma-Dibb RG. Nd:YAG laser in occlusal caries prevention of primary teeth: A randomized clinical trial. Lasers Med Sci. 2015;30(2):761-768. doi:10.1007/s10103-013-1417-z.

28. Hossain M, Kimura Y, Nakamura Y, Yamada Y, Kinoshita JI, MatsumotoK. A study on acquired acid resistance of enamel and dentin irradiated by Er,Cr:YSGG laser. J Clin Laser Med Surg. 2001;19(3):159-163.

29. Fox JL, Yu D, Otsuka M, Higuchi WI, Wong J, Powell G. Combined effects of laser irradiation and chemical inhibitors on the dissolution of dental enamel. Caries Res. 1992;26(5):333-339.

30. Tagliaferro EP, Rodrigues LK, Nobre Dos Santos M, Soares LE, Martin AA. Combined effects of carbon dioxide laser and fluoride on demineralized primary enamel: an in vitro study. Caries Res. 2007;41(1):74-76.

31. Bevilácqua FM, Zezell DM, Magnani R, da Ana PA, Eduardo Cde P. Fluoride uptake and acid resistanceof enamel irradiated with Er:YAG laser. Lasers Med Sci. 2008;23(2):141-147. doi:10.1007/s10103-007-0466-6.

32. Kwon YH, Lee JS, Choi YH, Lee JM, Song KB. Change of enamel after Er:YAG and CO2 laser irradiation and fluoride treatment. Photomed Laser Surg. 2005;23(4):38994. doi:10.1089/pho.2005.23.389.

33. Azevedo DT, Faraoni-Romano JJ, Derceli Jdos R, PalmaDibb RG. Effect of Nd:YAG laser combined with fluoride on the prevention of primary tooth enamel demineralization. Braz Dent J. 2012;23(2):104-109.

34. Chin-Ying SH, Xiaoli G, Jisheng P, Wefel JS. Effects of CO2 laser on fluoride uptake in enamel. J Dent. 2004;32(2):161167. 